\title{
Perspectives on Spinal Dysraphism : Past, Present, and Future
}

\author{
Dachling Pang ${ }^{1,2}$ \\ Department of Paediatric Neurosurgery, Great Ormond Street Hospital for Children, NHS Trust, London, UK \\ Department of Paediatric Neurosurgery, ${ }^{2}$ University of California, Davis, CA, USA
}

This article recounts the author's personal views and recollections of the history, conception of embryogenetic theories, application of intraoperative electrophysiology, and development of prospective treatment recommendations of several important spinal dysraphic malformations, including limited dorsal myeloschisis, dermal sinus tract, retained medullary cord, terminal myelocystocoele, and complex spinal cord lipomas.

Key Words : Spinal dysraphism · Embryology.

\section{INTRODUCTION}

Risking being labelled a repetitious drudge, I nevertheless feel compelled to begin this essay with total resection of complex spinal cord lipomas, because since the results of this work appeared during the cusp of 2009 and $2010^{11,12)}$, the "landscape" of spinal dysraphic surgery has gradually undergone a seismic change. Not only are paediatric neurosurgeons approaching lipomas with a new eye, while slowly wiping off the "old" smear of partial resection, people during the current decade are seemingly more aware of other less common categories of spinal dysraphic malformations such as limited dorsal myeloschisis (LDM), retained medullary cord (RMC), and terminal myelocystocoele (TMC). And I see no better way to start than recounting a brief history of the technique of total

\section{lipoma resection.}

When I began my academic career as faculty at the University of Pittsburgh in 1979, I started treating lipomas with partial resection in precisely the way drilled into my consciousness during my training: "Always skim off the top of the lipoma, never get near the spinal cord or you will paralyse the patient, and close the dura primarily as best you can." In other words, do a partial resection. In the summer of 1991, I tallied up the results of my own partial resection series and found that 76 of 116 patients came back with recurrent symptoms of re-tethering, often of agonising and debilitating nature, worse than the initial complaints ${ }^{12}$. It was then obvious that the old way of treatment was ineffective. I was therefore severely tempted to test the hypotheses that partial resection maintains the tethering state via the bulky remainder fibrofatty slab with

\footnotetext{
- Received : January 25, 2020 •Revised : April 28, 2020 •Accepted : April 28, 2020

- Address for reprints : Dachling Pang

Department of Paediatric Neurosurgery, Great Ormond Street Hospital for Children, NHS Trust, Great Ormond Street, London, WC1N3JH, UK Tel : +44-20 7405 9200, Fax : +44-20 7813 8279, E-mail : pangtv@aol.com, ORCID : https://orcid.org/0000-0002-6603-6546
} 
new adhesions to the tight dural closure, and that complete resection of the fat without chafing the cord (if possible), piato-pia roll-up closure of the well-trimmed neural placode, and generous graft closure of the overlying dura, would create a much more commodious environment for the radically reconstructed placode, ultimately making it less conducive to re-tethering.

I did test these hypotheses in July of 1991, often with unrepeatable emissions of fear. The first batch of total resection cases were done with comparatively primitive intraoperative electrophysiology monitoring, but I very quickly realised that total resection of fat and radical reconstruction of the placode were indeed feasible and safe, with no observable ill-effects on follow-up. After a few heart-pounding plunges seemingly straight into the spinal cord at the rostral edge of the lipoma in our early cases, I discovered the now-familiar but erstwhile deemed mystical "white plane", which in reality is no more than a thin netting of fibrous strands conveniently separating fat from neural tissue, just sturdy enough to allow the dissecting scissors to glide over during the entire subsequent purchase. I soon realised that this fibrous netting is present in every single lipoma regardless of type and size, and can be traced and navigated on reliably from rostral to caudal and side to side over the entire lipoma-cord interface, irrespective of the orientation of this interface, and whether the interface is flat or undulating ${ }^{11)}$. Discovering this white plane is of monumental importance in our pursuit because identifying it and respecting its integrity guarantees safety to the underlying spinal cord and has remained the absolute key to successful total resection of even the most appallingly huge lipomas with fearsome overhanging promontories of fat.

Another early battle was finding the right graft material and developing the best technique of closure. My colleagues and I quickly discarded autologous fascia lata because it is too soft and tends to stick to the neural placode even during normal inspiration, thus negating the purpose of making a capacious chamber. We then tried different artificial materials such as Gortex, latex, and silastic sheets but found all of them either too stiff or having unacceptable cerebrospinal fluid (CSF) leakage through the needle holes. We finally settled on chemically treated bovine pericardial patch (Duroguard) which is soft enough to be compatible with infant dura and without CSF leak. We also found that it is vital to match the graft size and shape with careful measurements to the contemplated configuration of the new dural sac, and line up the circumference of the graft with the rim of the dural defect using interrupted 4-0 nylon or prolene sutures, before putting in the definitive running 5-0 prolene suture to achieve absolute watertight closure. This careful graft-to-defect matching eliminates inward folding of the graft that could potentially press against the placode surface ${ }^{11}$.

The rest of this arduous travail is, of course, history. Our long term results of total resections of all complex lipoma types including redo lesions, first published in 2010 of 218 cas$\mathrm{es}^{12)}$ and then of 315 cases published in $2013^{15)}$ show a progression-free survival (PFS) of $88.5 \%$ stretched over 21 years, versus $34.5 \%$ for our own partial resection series over 11 years. If just the virgin (previously unoperated) lipomas are considered, the PFS goes over $95 \%{ }^{15)}$. The overwhelming advantage of total over partial resection is no longer in doubt. And if only the asymptomatic young children with virgin lipomas are selected, their long term PFS reaches a stunning 98.8\% ${ }^{15}$, making an indisputable case for prophylactic treatment for asymptomatic children with total resection, who otherwise suffer the fate of spontaneous deterioration without surgery in at least $40 \%$ of cases over their long life spans. Having made that overarching recommendation of prophylactic surgery for all lipomas in 2010, I have since amended our initial stance on the small subgroup of chaotic lipomas, which turned out to have a much poorer outcome than the dorsal and transitional lesions. I now endorse waiting for symptoms to appear before attempting radical surgery for chaotic lipomas ${ }^{6}$.

My last comment regarding total lipoma resection has to do with the negative impression expressed by the general community of paediatric neurosurgeons shortly after our first publication on the subject in $2009^{11)}$ who believed the technique of safe total resection was pure wizardry that was practically unlearnable except by a few. Many colleagues have since come to observe the operation, and all are convinced that the technique, other than a few novel but easily learned tricks (such as finding the proverbial white plane), is no more than variations of basic microneurosurgical techniques any trained neurosurgeon can master. These former doubters but now newly invested envoys have since set up their own practice of this technique in different parts of the world, rapidly scaling their respective learning curves, and are dutifully compiling their own series of total lipoma resection ${ }^{6}$. 


\section{GASTRULATION DEFECTS AND SPLIT CORD MALFORMATION}

In 1992, my colleagues and I published our series of split cord malformation and introduced our unified theory of embryogenesis of all double spinal cord malformations ${ }^{5,7)}$. We knew then there had to be a conduit communicating between the yolk sac and the amniotic sac during gastrulation to allow for endoderm to migrate up into the midline tract that divides the proto-notochord and the neural plate in halves, around which all the other primitive structural stem cells cluster, to ultimately develop into the heterogeneous features of a mature split cord malformation (SCM). We postulated initially the existence of an "accessory neurenteric canal" rostral to the primitive neurenteric canal normally at Hensen's node, which lies too caudal to the neural plate to explain SCM. During the last 15 years, we have come to think that this midline conduit we initially called "accessory neurenteric canal" in 1992, may actually be created by a focal and square-pulse type failure of midline integration of the converging proto-notochordal cells arising from both sides of the dorsal lips of Hensen's node ${ }^{8)}$. From thence, these cells normally pry their way in between the adjacent ectodermal-endodermal plates to integrate in the midline some distance rostral to Hensen's node, before joining the elongating rear of the midline notochord. At this focal region of failed midline integration of the notochord, each hemi-notochord now individually induces the overlying neural plate to form two hemicords, creating the eponymous features of the SCM. In between the hemicords, the ectoderm and endoderm plates remain coapted, allowing both germ elements and the surrounding mesodermal mesenchyme to migrate freely within this midline conduit which we called the ectoendomesenchymal tract. Depending on availability of the proper inducer molecules, these primitive cells from all three embryonic germ layers then form weird and discordant constituents adorning the hemicords and the median cleft in between, such as neurenteric and dermoid cysts, bone spurs, dural sheets, cartilage, fat, exuberant vasculature, and even nerve roots and ganglion cells from entrapped neural crest cells. The neurosurgical treatment of both type I and type II SCMs is more about eliminating these meddlesome and harmful elements than dealing with the split cords themselves.

\section{PRIMARY NEURULATION DEFECTS : LDM AND DERMAL SINUS TRACT}

The entity LDM, introduced in our 2010 publication ${ }^{13)}$, is now well-known and easily recognisable clinically by its characteristic skin crater with a squamous epithelial rather than skin base, and by its fibroneural stalk stretching from the base of the skin crater to the dorsal midline of the spinal cord readily demonstrable by magnetic resonance imaging (MRI) ${ }^{14}$. What counts as a fresh perspective for LDM is a new awareness of its frequent association with dermal sinus tracts and dermoid elements, often well hidden from imaging detection and even at surgery, posing a previously unrecognised danger of delayed occurrence of compressive dermoid cysts if part of the LDM stalk containing dermoid clusters is accidentally or intentionally left behind ${ }^{3}$. We have always known that both LDM and dermal sinus tract are the end results of incomplete disjunction of the cutaneous- and neuroectoderms probably due to focal failure of dorsal fusion of the neural plates. The persistent tissue link, or stalk, between the future skin and the subjacent spinal cord can be predominantly neural or squamous epithelium, resulting in the LDM or dermal sinus, respectively. We have seen well-displayed parallel LDM fibroneural stalk and complete dermal sinus tract adjacent to each other from the same skin crater to neighbouring points on the dorsal spinal cord, as well as in-tandem sinus tract and fibroneural stalk in a single stretch, prompting clean resection of both stalks at surgery to complete the untethering process ${ }^{14}$. What we did not know are the not-so-uncommon presence of deeply embedded squamous epithelial nests within an otherwise typical velvety-looking LDM stalk ${ }^{3}$. If the surgeon simply detaches the LDM's insertion on the spinal cord just to untether the latter, and leaves behind part of the innocuous looking LDM stalk hoping to minimise the extent of laminectomy, the hidden dermoid nests within the remainder stalk may well grow into large, compressive dermoid cysts years later to unhappy consequences. We have recently updated several of such cases, some of which were results of our own ignorance $^{3)}$. In our recent series of over 70 cases of LDM, over 10\% have proven to harbour some form of dermoid elements, prompting our recent stern recommendation of complete resection of the LDM stalk from skin to cord in every case, regardless of length and extent of bony exposure ${ }^{14,16)}$. 


\section{SECONDARY NEURULATION DEFECTS : RMC AND TMC}

My first encounter with what we subsequently called RMC was in the 1980s, in a young girl with leg weakness whose MRI showed a thick neural structure with signal characteristics of spinal cord but which was so long that it appeared coextensive with the vertebral column down to the coccyx. This cord-like structure also had a hydromyelic centre. I did not know what it was then, but have since seen many cases of similar elongated cord-like extension of the conus. With advent of the new knowledge on the morphological stages of secondary neurulation, it occurred to me that this odd thing could very well be an arrested state of mid-secondary neurulation when apoptosis stopped abruptly at the stage of the cavitated medullary cord. The final stage of programmed destruction of most of this medullary cord to form the filum terminale is therefore missing, and the thick medullary cord remains ${ }^{10)}$. Cord tethering was due to pulling by this monstrous trunk of redundant non-functional neural tissue, and treatment was predicated on finding the junctional boundary between the functional conus above and the non-functional trunk below using intraoperative electrophysiology, and then cutting away the medullary cord at the boundary. Histological examination of these resected specimens confirmed the presence of disorganised neurons, glial tissue, ependyma, and peripheral nerves, as expected within an unconsummated would-be spinal cord. RMCs are now well-recognised as a separate entity from what used to be thought an extraordinarily stout filum terminale.

A logical extension of the embryogenetic theory for RMC was postulated by us in 2012 to explain $\mathrm{TMC}^{9)}$. I called TMC a biological leviathan in our initial publication because of the almost mystical characterisations of its bizarre structure and its "unknowable" embryological origin in prior reports, which were legion but singularly unhelpful. I owe much of our new embryogenetic hypothesis of TMC to the laboratory work from Seoul National University led by Professors K. C. Wang and J. Y. Lee, who discovered the "terminal balloon" in chick embryos, which is a myelocystic distention of the terminal canal of the secondary neural tube during a transient phase at end-stage secondary neurulation, just before wholesale apoptotic dismantling of the caudal secondary neural tube ${ }^{4,17)}$. Longitudinal sections of these chick embryos at the terminal balloon stage bear an eerie resemblance to the human homologue of TMC, which thus inspired the epiphany that TMC could in fact be caused by an abrupt arrest of apoptosis during the brief terminal balloon stage, assuming this terminal balloon also exists in human secondary development.

Aside from endlessly contemplating on the fate of this terminal balloon, we also learned from our operative experience of over 40 cases of TMC that all TMCs, regardless of size, symmetry and complexity, are configured like an extruded trumpet of spinal cord tissue through a large dorsal bony defect, consisting of a functional proximal part endowed with live nerve roots, and a distal non-functional part composed of chaotic internal architecture. The key to successful repair of TMC will thus depend on locating the junctional boundary between functional and non-functional parts of the trumpet, much like finding the boundary between the "real" conus and defunct secondary neural tube in RMC, then make a clean cut along this boundary, discard the cumbersome bottom, neurulate the mouth of the proximal trumpet, and shove the greatly reduced bulk back into the spinal canal. Good result, then, is not so much resting on the demonic skill of the surgeon but on the competency of the electrophysiologist in accurately demarcating the functional from non-functional demesnes on the homogeneous looking neural structure, which leads us to the next, probably most important topic of intraoperative neurophysiological monitoring (IONM) in spinal dysraphism surgery.

\section{INTRAOPERATIVE NEUROPHYSIOLOGY MONI- TORING}

When one hears about intraoperative "monitoring", one is assuming that the test in question is being run continuously in real time as the procedure is being performed, to gauge any instantaneous changes in function. This is not how IONM is used in spinal dysraphism surgery. Because transcranial motor cortex stimulation (in transcranial motor evoked potential measurement [TcMEP]) produces significant jerking of all the muscles in the body, it cannot be executed while the actual dissection is being done. The usual practice is for the surgeon to call for a run after a particularly delicate or risky dissection has just been done, to check for adverse changes in the evoked motor potentials. Any changes would thus be "after the fact". 
The electrophysiologist also cannot run the test randomly; the two activities must be coordinated. Most importantly, the adverse change, when discovered, could have been produced by the surgical manipulation 10 or 15 minutes before. At this point, the best the surgeon could do is to halt the dissection until, hopefully, the loss of motor potentials has recovered, before undertaking further assault on the cord. Also, the routine continuous run of somatosensory evoked potentials (SSEP) is irrelevant in most tethered cord operations because the somatic sensory inputs for the SSEP are introduced through the $\mathrm{L}_{5}$ and $\mathrm{S}_{1}$ segmental roots, above most conus lesions involving mainly the $\mathrm{S} 2-\mathrm{S} 4$ spinal cord segments. Thus, neither TcMEP nor SSEP are ideal "monitoring modalities" for dysraphism surgery. However, evoked motor potentials monitoring through direct stimulation on real or suspicious neural structures using bipolar electrodes is sine qua non in dysraphism surgery. Direct contact with the stimulation probe using graded current intensities on nerve roots, suspicious bands, fibroneural globs, and other bewildering tissue muck frequently encountered in lipoma surgery, is often the only way to separate "live" roots from fibrous bands or defunct "dead" or vestigial nerves, and functional neural tissue from fibrous waste. Also, the anatomical reference levels of spinal cord segments and nerve roots are often "displaced" by abnormal development so that their correct identification is only possible through functional evaluation with the probe. As mentioned above, neither RMC nor TMC could be safely repaired without proper IONM.

There is currently still doubts amongst some physiologists about using TcMEP on newborns and young infants for fear of inducing seizures. The readers should be reassured that I have personally conducted TcMEPs on numerous new born infants with the approved voltage limits without ever causing seizures, and that it is a safe test for children of all ages. It is certainly true that for young infants, the minimum effective voltage has to be increased to compensate for poor myelination, and that the threshold for readable potentials is also more sensitive to inhalation anaesthetics, but these parameter adjustments can be made without compromising results. These details are listed in the lipoma chapter of this monograph.

One last point should be made about the electrophysiological version of the bulbocavernosus reflex (BCR). The electrical BCR circuit used in IONM involves the $\mathrm{S}_{2}-\mathrm{S}_{4}$ somatic sensory afferents from the perineal skin and the somatic $S_{2}-S_{4}$ efferent outputs to the voluntary external anal sphincter, which is distinct from the bladder-detrusor voiding circuit involving autonomic afferents from the bladder wall stretch receptors and the involuntary parasympathetic motor neurons to the involuntary bladder detrusor muscles. The two circuits are located within the same sacral cord segments $\left(\mathrm{S}_{2}-\mathrm{S}_{4}\right)$ but utilise different pathways within the cord. Thus, it is theoretically possible to injure one while preserving the other, and in reality probably does happen in some cases ${ }^{6}$. We have certainly seen cases of complete loss of the BCR at the end of surgery but no loss of micturition function in the patient. Also, it is not uncommon to lose the BCR on one side after lipoma resection, while the TcMEP to the anal sphincter remains intact, with variable changes in post-operative micturition. Thus, the correlation between the different patterns of BCR and anal sphincter TcMEP alterations and the actual resultant micturition and storage functions of the bladder, i.e., the practical value of BCR monitoring, is yet to be determined by a large prospective study.

\section{UROLOGICAL EVALUATION IN SPINAL DYSRA- PHISM}

While on the subject of ancillary testing, I must emphasise the importance of obtaining detailed urological evaluation of bladder function in children with complex spinal dysraphism, both before repair and serially as a follow-up tool. I like first to debunk the misconception that performing proper urodynamics in infants and young children has no value because they are below the age of cooperation. In reality, the key features we look for in a formal urodynamics study are uninhibited and hyperactive detrusor contractions indicative of an irritable bladder, vesicular capacity (a diminished capacity is seen with hyperactive detrusor, and an abnormally large capacity may indicate an atonic bladder) and compliance (poor compliance is seen with hypertrophic or even fibrotic bladder wall from chronic infections), post-void residual volume, and detrusor-sphincter dyssynergia (DSD), none of which needs cooperation from the patient ${ }^{6)}$. Ureteral reflux is an early warning for pyelonephritis and impending renal damage, and may be inferred from a distended upper urinary tract on ultrasound, but grade 1 reflux does not cause ureteral ectasia and its disclosure therefore requires a proper voiding cysto- 
urethrogram, which can be done at the same time as the urodynamics study by introducing contrast through the urinary catheter. The presence of any reflux, coupled with DSD, unequivocally identifies a hostile bladder potentially damaging to the kidneys, and treatment of the cause by untethering of the conus becomes much more urgent. As well, more aggressive management of the neuropathic bladder itself such as immediately instituting clean intermittent catheterisation (CIC), starting parasympatholytic drugs to lessen detrusor irritability, or even contemplating bladder augmentation if more conservative measures fail to curb reflux.

\section{JUNCTIONAL NEURULATION DEFECT}

Many years ago, I was sent a child from Mainland China who had practically normal leg functions but was completely insensate in the bladder and incontinent of stool and urine. His MRI showed a bizarre picture of an "upper conus" at $\mathrm{L}_{1}$ but a "lower conus" at $S_{2}$ connected to each other by a slender strand. I thought, erroneously as it turned out, he had a RMC, explored both structures, and found that the "upper conus" was in fact the terminal primary neural tube, whereas the bottom structure was a completely formed "true" conus with staunchly functional anal sphincter roots on stimulation. The primary cord above also stimulated credibly for feet and leg muscle action, but it was obvious the upper and lower cords were not connected functionally despite the intervening strand, which was totally inert on stimulation. The fact that both the primary and secondary neural tubes were fully formed locally yet neither anatomically nor functionally connected was amply verified by perfectly normal BCRs for the conus but complete absence of anal sphincter response with TcMEP. This and the essentially normal leg muscles responses by transcranial stimulation means the primary neural tube was wired for cortical control but the conus was not ${ }^{2}$.

My colleagues and I have since encountered three more patients with the same clinical profiles and almost identical MRI pictures, i.e., their caudally located secondary neural cords were separated from the upper primary spinal cords by 5 to 10 $\mathrm{cm}$, linked only by a puny strand. This anomaly had never been described before but we thought that the embryogenetic error had to have occurred during what Dady et al. ${ }^{1)}$ termed junctional neurulation in chick embryos, i.e., between the end of primary neurulation and the beginning of secondary neurulation. Our theory goes that the "unjoining" of the terminal primary neural tube from the caudally situated secondary neural tube, each well developed independently, can be caused by a mutation of the Prickle-1 gene within the planar cell polarity (PCP) pathway during this junctional period, causing a disruption of the maintenance of continuity between the two neural tubes. We called this anomaly Junctional Neural Tube Defect (JNTD) in its first appearance in print in $2017^{2)}$. It is interesting that at least three other similar cases have since been reported by other neurosurgical units.

\section{CONCLUSION : CHICK OR MOUSE?}

This brings us to the intriguing and ever-entertaining question of whether human neurulation mimics the chick or the murine model, each popular with its respective school of experimental embryologists. There are, deus scit, hordes of stern adherents and defenders for each model, all armed with sworn arguments, but I tend strongly to subscribe to the chick, based on three observations. First, the JNTD example I cited above can only be possible if human junctional neurulation copies the chick's because the murine secondary neural tube extends uninterrupted from the caudal terminus of the primary neural tube and is therefore unlikely to be fully formed locally but disenfranchised from the rest of the neural tube, as is possible in avian junctional neurulation, where neural progenitor cells for the future conus more or less develop in situ separate from the primary neural tube $e^{2)}$. Second, the terminal balloon mentioned in the embryogenesis of TMC has only been seen in chick embryos but not in the mouse ${ }^{9)}$. Lastly, we have regularly counted two or three lumina within the RMC specimens resected from humans, matching the multiple lumina in the chick medullary cord and not the single lumen within the murine medullary cord ${ }^{10)}$.

Hence, my stand is with the chick.

\section{CONFLICTS OF INTEREST}

No potential conflict of interest relevant to this article was reported. 


\section{INFORMED CONSENT}

This type of study does not require informed consent.

\section{AUTHOR CONTRIBUTIONS}

\author{
Conceptualization : DP \\ Data curation : DP \\ Methodology : DP \\ Visualization : DP \\ Writing - original draft : DP \\ Writing - review \& editing: DP
}

\section{ORCID}

Dachling Pang https://orcid.org/0000-0002-6603-6546

\section{References}

1. Dady A, Havis E, Escriou V, Catala M, Duband JL : Junctional neurulation: a unique developmental program shaping a discrete region of the spinal cord highly susceptible to neural tube defects. J Neurosci 34 : 13208-13221, 2014

2. Eibach S, Moes G, Hou YJ, Zovickian J, Pang D : Unjoined primary and secondary neural tubes: junctional neural tube defect, a new form of spinal dysraphism caused by disturbance of junctional neurulation. Child Nerv Sys 33 : 1633-1647, 2017

3. Eibach S, Moes G, Zovickian J, Pang D : Limited dorsal myeloschisis and associated dermoid elements. Child Nerv Sys 33 : 55-67, 2017

4. Lee JY, Kim SP, Kim SW, Park SH, Choi JW, Phi JH, et al. : Pathoembryogenesis of terminal myelocystocele: terminal balloon in secondary neurulation of the chick embryo. Child Nerv Sys 29 : 1683-1688, 2013

5. Pang D : Split cord malformation: part II: clinical syndrome. Neurosurgery $31:$ 481-500, 1992
6. Pang D : Surgical management of complex spinal cord lipomas: how, why, and when to operate. A Review. J Neurosurg Pediatr 23 : $537-$ 556, 2019

7. Pang D, Dias MS, Ahab-Barmada M : Split cord malformation: part I: a unified theory of embryogenesis for double spinal cord malformations. Neurosurgery $31:$ 451-480, 1992

8. Pang D, Hou YJ, Wong ST : Classification of spinal dysraphic malformations according to embryogenesis: gastrulation defects and split cord malformation in DiRocco C, Pang D, Rutka J (eds) : Textbook of Paediatric Neurosurgery. Springer Publishers : Cham

9. Pang D, Zovickian J, Lee JY, Moes GS, Wang KC : Terminal myelocystocele: surgical observations and theory of embryogenesis. Neurosurgery 70 : 1383-1405; discussion 1404-1405, 2012

10. Pang D, Zovickian JG, Moes GS : Retained medullary cord in humans: late arrest of secondary neurulation. Neurosurgery 68 : 1500-1519; discussion 1519, 2011

11. Pang D, Zovickian J, Oviedo A : Long-term outcome of total and neartotal resection of spinal cord lipomas and radical reconstruction of the neural placode: part I-surgical technique. Neurosurgery 65 : 511-528; discussion 528-529, 2009

12. Pang D, Zovickian J, Oviedo A : Long-term outcome of total and neartotal resection of spinal cord lipomas and radical reconstruction of the neural placode, part II: outcome analysis and preoperative profiling. Neurosurgery 66 : 253-273; discussion 272-273, 2010

13. Pang D, Zovickian J, Oviedo A, Moes GS : Limited dorsal myeloschisis: a distinctive clinicopathological entity. Neurosurgery 67 : 1555-1579; discussion 1579-1580, 2010

14. Pang D, Zovickian J, Wong ST, Hou YJ, Moes GS : Limited dorsal myeloschisis: a not-so-rare form of primary neurulation defect. Childs Nerv Syst 29 : 1459-1484, 2013

15. Pang D, Zovickian J, Wong ST, Hou YJ, Moes GS : Surgical treatment of complex spinal cord lipomas. Childs Nerv Syst 29 : 1485-1513, 2013

16. Wong ST, Kan A, Pang D : Limited dorsal spinal nondisjunctional disorders: Limited dorsal myeloschisis, Congenital spinal dermal sinus tract, and mixed lesions in DiRocco C, Pang D, Rutka J (eds) : Textbook of Paediatric Neurosurgery. Springer Publishers : Cham

17. Yang HJ, Wang KC, Chi JG, Lee MS, Lee YJ, Kim SK, et al. : Neural differentiation of caudal cell mass (secondary neurulation) in chick embryos: Hamburger and Hamilton stages 16-45. Brain Res Dev Brain Res $142: 31-36,2003$ 